\begin{tabular}{|c|c|}
\hline Title & Measuring W ork Factors: A Case Study to I dentify Relationships among W ork A ctivities, Styles and an Environment \\
\hline Author(s) & Sakamaki, Motohiro; Ikeda, Fumihito; Takada, Shingo; Nakakoji, Kumiyo \\
\hline Citation & $\begin{array}{l}\text { Lecture Notes in Computer Science, 1670, 164-176 } \\
\text { https://doi.org/10.1007/10705432_15 }\end{array}$ \\
\hline Issue Date & 1999 \\
\hline Doc URL & http:/hdl.handle.net/2115/49215 \\
\hline Rights & The original publication is available at www.springerlink.com \\
\hline Type & article (author version) \\
\hline Note & $\begin{array}{l}\text { Second International Workshopon Cooperative Buildings: Integrating Information, Organizations and A rchitecture } \\
\text { (CoBuild' 99) }\end{array}$ \\
\hline File Information & CoBuild99_Submit.pdf \\
\hline
\end{tabular}

Instructions for use 


\title{
Measuring work factors: A case study to identify relationships among work activities, styles and an environment
}

\author{
Motohiro Sakamaki(1), Fumihito Ikeda(1)(2), \\ Shingo Takada ${ }^{(3)}$, Kumiyo Nakakoji(2)(4)(5) \\ (1) Research and Development Headquarters, NTT DATA Corporation \\ (2) Graduate School of Information Science, Nara Institute of Science and Technology, Japan \\ (3) Faculty of Science and Technology, Keio University, Japan \\ (4) Software Engineering Laboratory, SRA Japan \\ (5) PRESTO, Japan Science and Technology Corporation \\ \{samakaki, fumi\}@rd.nttdata.co.jp,michigan@doi.cs.keio.ac.jp, kumiyo@is.aist-nara.ac.jp
}

\begin{abstract}
A work environment needs to be designed based on the nature and characteristics of the work activities and the work styles. Unless we have a clear understanding of what factors of work activities and styles depend on what factors of work environments and vice versa, we would not be able to modify or add components of work environments "effectively." This paper first presents our framework to measure work factors of work activities, styles and an environment, and then discusses results of our case study of applying the framework to actual work practice at NTT Data Corporation. Our framework consists of three representational models: the KPM (Knowledge Production Model) for representing work activities, the MS-GI (Mobile-Settled, Group-Individual) model for characterizing work styles, and the SOU (System-Office-Usage) model for representing a work environment. We have conducted a survey on 274 workers at an organizational unit of NTT Data Corporation by applying the three models. The result shows that the framework is successfully applied to understand the relationships among the three work factors at least partially if not completely. The paper concludes with a discussion of how the framework can be used to evolve work environments by taking into account work activities and styles.
\end{abstract}

Keywords work styles, work environments, measurement, case studies, office design

\section{Introduction}

Work environments need to be designed based on the working style of people who use the environments [1][2]. A poor fit between working styles and the work environment can result in reduced productivity[2]. Therefore, the design of work environments to enhance quality and output must take into account characteristics of work activities, characteristics of working styles, and the interaction of these factors.

With this fact quite well-shared among communities, however, there has been little research on how to represent these factors; what types of work activities are performed and how they can be classified, what working styles exist and how they can be captured, what constitutes a work environment and how it should be represented, and finally and most interestingly, how these factors depend on one another.

This paper first presents our framework to quantitatively measure work factors including work activities, styles and an environment, and then discuss results of our case study that has applied the framework to actual work practice and construction of an office at NTT Data Corporation. Our framework consists of three representational models: the KPM (Knowledge Production Model) for representing work activities, the MS-GI (Mobile-Settled, GroupIndividual) model for characterizing work styles, and the SOU (System-Office-Usage) model for representing a work environment. The results show that the framework partially explained relationships among the three work factors in the NTT DATA Corporation setting.

\section{Representing work activities, styles and an environment}




\subsection{The framework}

On the basis on the background described above, our goal was to identify what work factors depend on what other work factors. Such understanding about the relationships among the factors will then guide us in designing and evolving work environments including both physical (office spaces) and logical (computer tools, work policies and rules) ones.

To identify the relationships among the factors, we have taken an approach to "measure" aspects of work-related factors. Representations for each of such aspects are thus developed to achieve the goal. Fig.1 illustrates our framework for representing work. We view "work" from three perspectives: work activities, work styles and work environments. Work activities represent what types of operations and functions people perform in their work. Our approach is to characterize work activities in terms of what "information and knowledge" is produced, shared and used. We propose the KPM (Knowledge Production Model) for representing work activities.

Work styles represent what work settings people are in during their work. The MS-GI(Mobile-Settle, GroupIndividual) model categorizes work styles in terms of two dimensions : whether people are mobile or settled, and whether people work within a group or independently.

Finally, work environments represent both the physical and logical surroundings of people in their work hours. The SOU (Systems-Office-Use) model is used to characterize a work environment. The system aspect include what types of computer tools exist in the environment. The office aspect illustrates how the physical office space is designed in the environment. The use aspect illustrates what rules and policies exist in the environment.

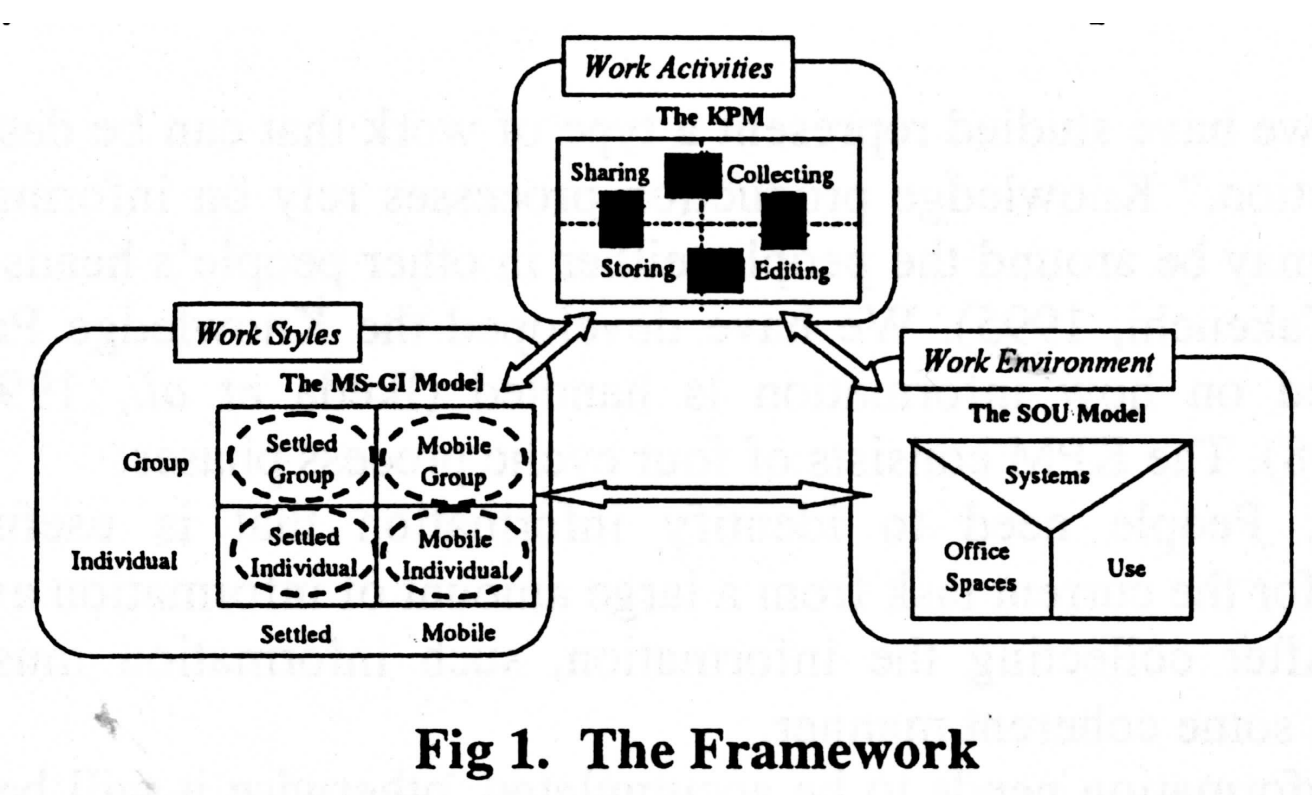

\subsection{Merit of using the framework}

Assuming the availability of data on the three work factors and the existence of "stable" relationships among these factors, it should be possible to use these data and relationships to guide the development of new work environments and to analyze the effect of additions to an existing work environment.

For example, when an organization builds a new work environment for a team, the organization can use the models as follows to: a) examine work styles of the team; b) identify what phase of the KPM people in each work style think is important in terms of the relation between work activities and work styles; and c) determine what elements should be incorporated in the new work environment. Similarly, when an organization adds a new element to an existing work environment(e.g., new computer system, new space, new rules), the organization can use the framework to: a) identify what phase of the KPM the introduced element supports in terms of the relation between work activities and work environment; b) examine what work style is important to the identified phase in terms of the rela- 
tion between work activities and work styles; c) identify work styles of the people in the team; and d) compare the work styles identified in a) and the work styles identified in b).

The remainder of this section describes each of the three models in more detail.

\subsection{Representation of work activities: The KPM}

The work activities we have studied represent a type of work that can be described as "Knowledge Production." Knowledge production processes rely on information that people have or that may be around the people, either in other people's heads or in the world [10]. We have developed the Knowledge Production Model (KPM) based on how information is handled [5][6][11]. The KPM consists of four cyclic process phases:

Collecting: People need to identify information that is useful and/or necessary for the current task from a large amount of information available.

Editing: After collecting the information, such information must be put together in some coherent manner.

Storing: Information needs to be accumulated, otherwise it will be lost and become unusable.

Sharing: Information needs to be shared among other people in the organization. "New" information needs to be shared among other people in the organization. "New" information provided by other people becomes a "seed" for creating new knowledge.

These four process phases may not be always distinct but may overlap. For example, one may become aware of the need for collecting more information while editing already collected information.

\subsection{Representation of work styles: MS-GI model}

We have characterized work styles in two dimensions. One is whether one performs "mobile" work or "settled" work. By "settled", we mean those who always work at fixed places. The other dimension characterizes people by whether they generally work as "individually-oriented" or as "group-oriented". Individually-oriented means work that can be conducted independent of others. Group-oriented people need to cooperate with each other for accomplishing their tasks. Looking at work styles from these two dimensions results in the four working styles: MobileGroup, Mobile-Individual, Settled-Group, and Settled-Individual.

\subsection{Representation of work environment: the SOU model}

We view a work environment from three perspectives: systems, physical office spaces, and how they are used [5] [6]. The systems perspective represents computer tools and systems that are incorporated in the environment. This aspect has long been pointed out as critical in various types of works [12] [9], and existing research has basically concentrated on this factor in dealing with work environments.

The office space perspective is how the physical space is used within the work environment. This has also been reported to affect our work productivity [8] [2]. The layouts of office cubicles as well as types of meeting spaces are examples of this perspective.

The use perspective denotes how the computer system and office space are used, including rules and policies governing people at an organization. As Grudin [4] pointed out that computer systems may benefit only a certain type of people without benefiting others, this factor is especially important in dealing with work environments.

\section{A Case Study: Measuring Work Factors}

This Section presents results of our case study that applied the above framework in the real work practice.

\subsection{Investigation Outline}


In order to verify whether each relationship among factors in the framework is measurable, we have conducted the following three questionnaires.

Investigation to capture important elements of current work environment and desired elements for future environment

Investigation to categorize the SOU elements to each phase of the KPM

Investigation to classify a worker's work styles

About questionnaire A) and C), we have conducted surveys on 274 members at NTT DATA Corporation, who are engaged in sales, accounting, general affairs, planning, system development, and research. The details of subjects are that 179 subjects belonged to software research institute and 95 subjects were belonged to the Kyushu branch. About questionnaire B), we have conducted surveys on only 30 members who have worked in the Distributed Cooperative model office(see 3.8) and who have engaged in software research and development. These 30 subjects are contained in 274 subjects.

In our case study, before our conducting surveys, we have first analyzed existing work environment in terms of the SOU model(see 3.2). We have identified elements for each of the three perspectives of the SOU model based on the $\mathrm{KPM}($ see 3.3). We then conducted a survey on what work styles those subjects were engaged in(questionnaire C), see 3.4). We have next asked each subject what elements of the work environments will be important currently and necessary for the future (questionnaire A)). Based on the sets of data collected in questionnaire A) and C), we have analyzed if there are relationships between work styles and characteristics of work environments(see 3.5). Then, in order to prove our hypothesis about the relationship between the SOU model and the KPM, we have conducted survey on what SOU elements was categorized in terms of what process of the KPM in questionnaire B)(see 3.3 and 3.6). At last, we have analyzed relationships between work activities and work styles based on the results from 3.3, 3.4 and 3.5(analysis result will show in 3.7).

The remainder of this section details the case study.

\subsection{Application of the SOU model}

In the analysis of the organization, we have identified thirty-one elements of computer systems, thirty-five elements of physical office space, and fifteen elements of practice (the detailed description of the elements are in [6]). Some of the elements are:

Systems:

electronic approval systems: ones that automatically identify and ask the appropriate person for approving a purchase using the organization's budget.

tele-conferencing systems: ones that allow meetings between people that are remotely located with TV screens showing views from each site.

Office spaces:

individual spaces: spaces that are segmented as booth so that people respect each other's privacy.

large meeting spaces: meeting spaces for 10 or more people.

Uses:

rules on managing information: rules instituted regarding management of information, such as how new information should be stored.

concentration rules: rules that help people to maintain concentration, for instance, one of them states that how one should let others know that he/she wants not to be disturbed.

We have asked each subject what elements of the work environments (1) are currently important for them and (2) will be important and necessary for the future on questionnaire A). By using this application and application for the MS-GI, we can compare the difference in the SOU elements for every work styles.

\subsection{Application of the KPM}

After we have identified the elements of the work environment as described above, we have categorized each element in terms of what process phase of the KPM the element supports. Some examples of the categorization include:

Tele-conferencing systems: collecting, editing and sharing (because such a system supports to communicate with each other, gain information from others and may produce some artifacts through communication) 
Library spaces: collecting, storing and sharing (because such a space store and share many information that can be shared among group members)

Detailed results of this survey can be found in [6]. This relationship between each elements and the KPM is only our hypothesis. In order to verify our hypothesis, we have conducted questionnaire B) which set up some routine questions by which the SOU elements are connected each process phase of the KPM. Some of the routine questions to all elements are:

Collecting: has it been become easy to retrieve information?

Editing: has it been become easy to come out of a new idea?

Storing: has it been become easy to recycle information or/and idea?

Sharing: has it been become easy to share information or /and idea?

By using this application, the KPM and the SOU model are related.

\subsection{Application of the MS-GI model}

In order to analyze what work style the subjects are categorized into, we have used questionnaire C) that contains ten questions each regarding the two perspectives: "mobile vs. settled (MS perspective)" and "group-oriented vs. individual-oriented (GI perspective)." Subjects were asked to select one of the four levels between the two extremes.

Examples of questions asked for the MS perspective include:

How standardized is your work?: (1) routine (2) non-routine

With whom do you work together?: (1) always with the same group of people (2) always different

Examples of questions regarding the GI perspective include:

How do you set up a goal?: (1) by coordinating within a group (2) by setting up individually

What is the purpose of storing information?: (1)mainly for myself (2) mainly for the group

Answers given to each of those questions are quantified and weighted sum were used to characterize each subject's work style (the algorithm used is detailed in [11]).

\subsection{Relationship between work styles and work environments}

From the result of questionnaires A) and C), we tried to clarify the relation between work styles and work environment in the framework. We supposed that each work styles needed each different elements of the SOU model. From the result of this investigation, we found that our hypothesis was filled mostly. The result of the survey is shown in Fig 2. In Fig 2, each SOU factors is taken along a vertical axis, and the work styles is taken along the horizontal axis. The cell which is gray is the element with which workers' need was accepted to be high. Hereafter, we will describe tendency about each work styles.

\section{SOU elements for Mobile-Group}

The need for elements of office is high

The need for informal communication is high

The need for individual concentration is not so high

The feature of this work style is that the need for System elements and Use elements is not so high. Instead, they had demanded elements for sharing information spatially, such as white board, pinup board and OHP screen. Conversely, in the same Office elements, the need for individual concentration, such as Cave, is low. From this result, they tend to seldom pay attention to individual environment, if the spatial environment for their collaboration is prepared. 
The need for the broad access to information is high

The need for flexible access to the received information is high

Their feature is not adhering to especially system elements and use elements but they want to access information broadly like $W W W$, subscription service, personal cellular phone, librarian support and so on. Moreover, as opposed to Mobile-Group, their needs to improve personal environment, such as ergonomics furniture, Cave, is also high.

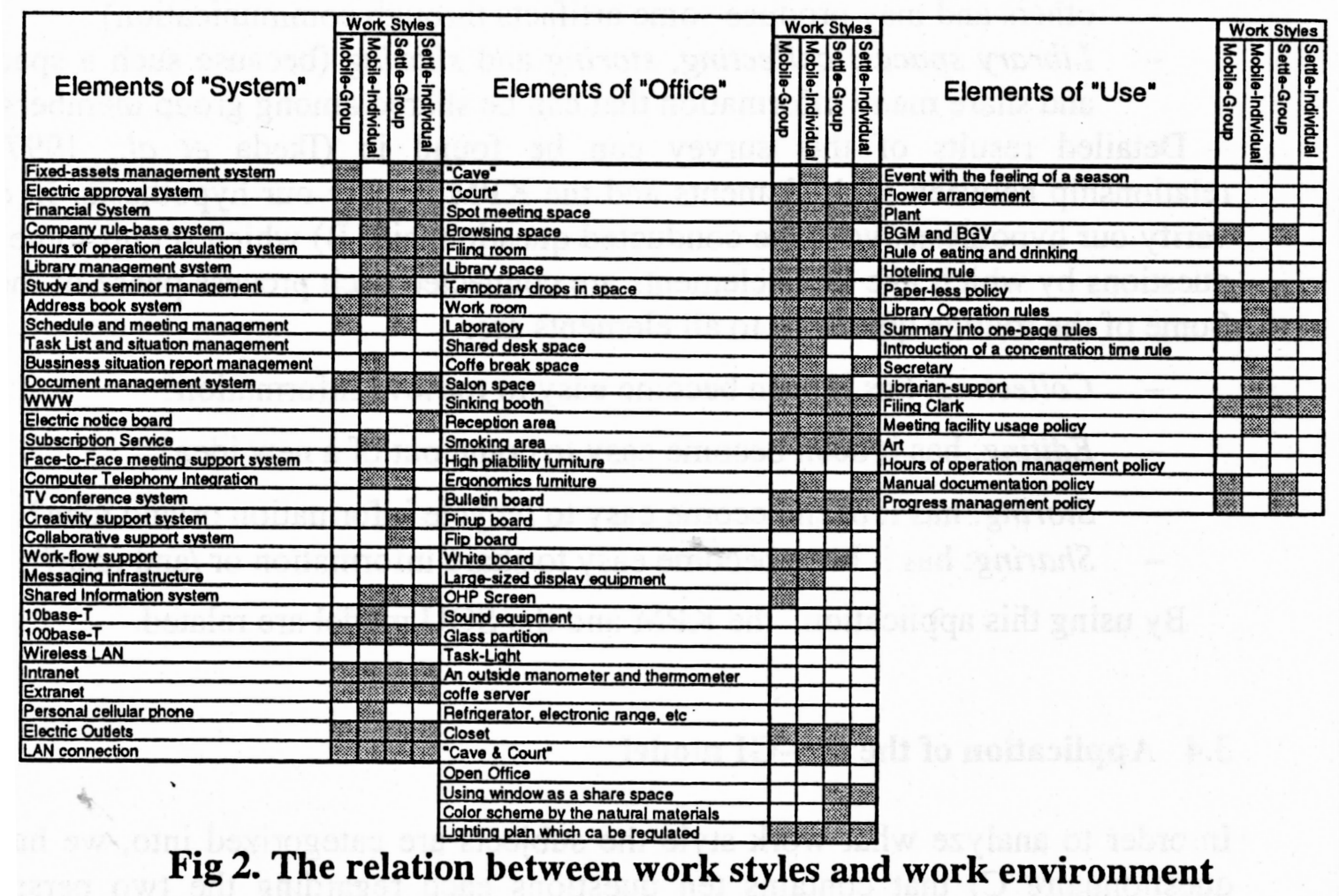

\section{SOU elements for Settle-Group}

The need for flexible access to the received information is high

This work style has tendency resembled Mobile-Individual very well. However, since the need for CTI, shared information system is high and the need for office elements, such as shared desk space and coffee break space, is conversely low, Settle-Group does not desire to communicate in real space but in virtual world. Moreover, the needs to creativity support and collaborative support were high. However, we have not expected at all about this tendency. So, we need to gaze about this point.

\section{SOU elements for Settle-Individual}

The need for accessing to analog data is very low

There is no factor of being high in this work style only. Conversely, there are many factors that only to this work style does not have but other work styles have. Their characteristic elements are in Office elements, such as browsing space, filing room, library space, salon space, white board and so on. Each of these are the elements for catching 
information in analog. From these things, they tends to dislike the access to analog data very much. This is known also from the need for electric notice board, shared information system being high.

\section{Categorization SOU elements based on WS-GI axis}

Next, let us consider about the tendency of SOU elements with Mobile-Settle or Group-Individual. The needs for "Group" are schedule and meeting management, pinup board, manual documentation policy, progress management policy. Conversely, the needs for "individual" are company rule-based system, Cave, and ergonomics furniture. From this result, the work style classification by "Group" and "Individual" shows that there is remarkable difference in the need for the SOU model.

On the other hand, there is no remarkable difference between "Mobile" and "Settle". What does this mean? As for this, we wonder if the axis of "Group-Individual" and the axis of "Mobile-Settle" do not cross at right angles. For this reason, it may be unsuitable to consider only these two axes as an factors for representing work styles.

\subsection{Relationship between work activities and work environments}

On the basis of the result of questionnaire B), we have arranged each the SOU elements to each phase of the KPM. In this investigation, we verified only about a part of the SOU model which has introduced into the model office(see 3.8). The result is shown in Fig 3. The feature of this mapping is that "Cave \& Court" covers the broad range of the KPM. "Cave \& Court" is the layout which arranges some individual work spaces(Cave) around centering on a meeting space(Court). Although "Cave \& Court" is classified into the elements of Office factor, it also covers the field of System and Use, such as "TV conference system", "electric white board", "meeting usage policy" and so on, broadly. This shows that in case we represent work environments, introducing SOU elements simultaneously is more effective rather than introducing SOU elements individually.

Since this mapping is the analysis only for about 30 subjects who are in the new office, it is dangerous to use this result as a general solution. However, we think that this case study has suggested that relating of the KPM and the SOU model is possible.

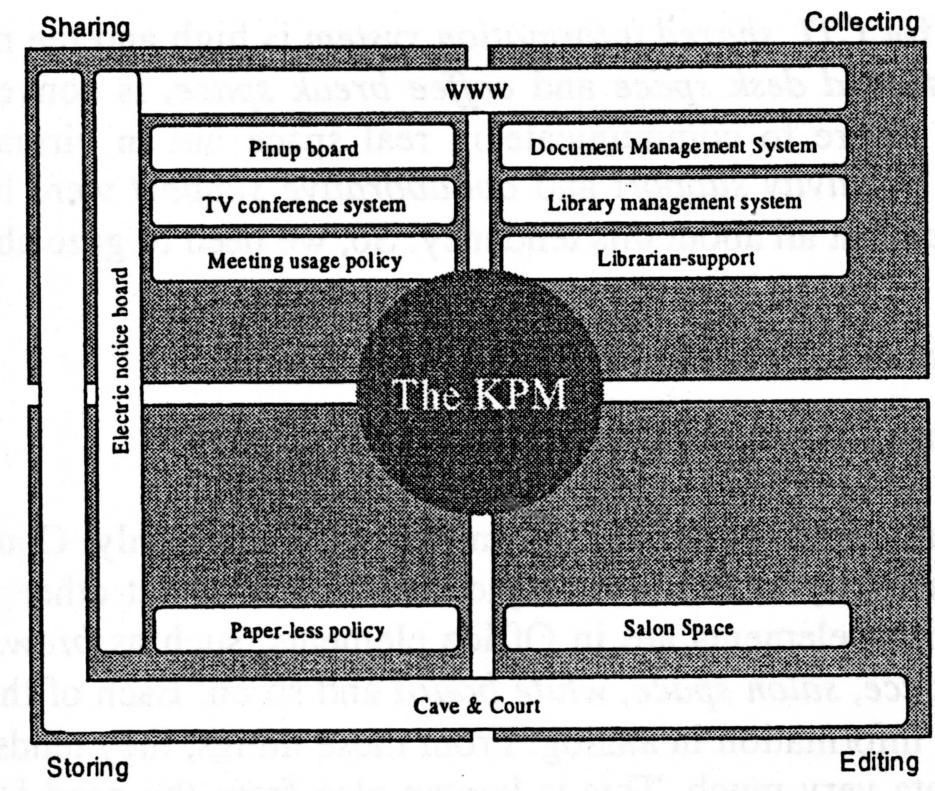

Fig 3. The relation between work activities and work environments

\subsection{Relationship between work activities and work styles}


In our case study, we have not conducted evaluation which connects work activities and work styles directly. Then, we tried to clarify the relation between the KPM and MS-GI model through the SOU model by using the measured results of questionnaire A), B) and C). At the beginning, in work style which intersects perpendicularly, we think respectively that completely reverse tendency should come out. The result is shown in Fig 4.

Consequently, we found the remarkable difference between "Mobile-Group" and "Settle-Individual". This was as our expected. However, between "Mobile-Individual" and "Settle-Group", we have not found the remarkable difference. From this result, as we described in section 3.5, we have to suspect the rectangular-cross of axis in MS-GI model. However, we think that clarifying the relation between the KPM and MS-GI model has been attained to some extent via the SOU model. Therefore, our conviction that a setup of the framework by representation of work activities is effective is confirmed.

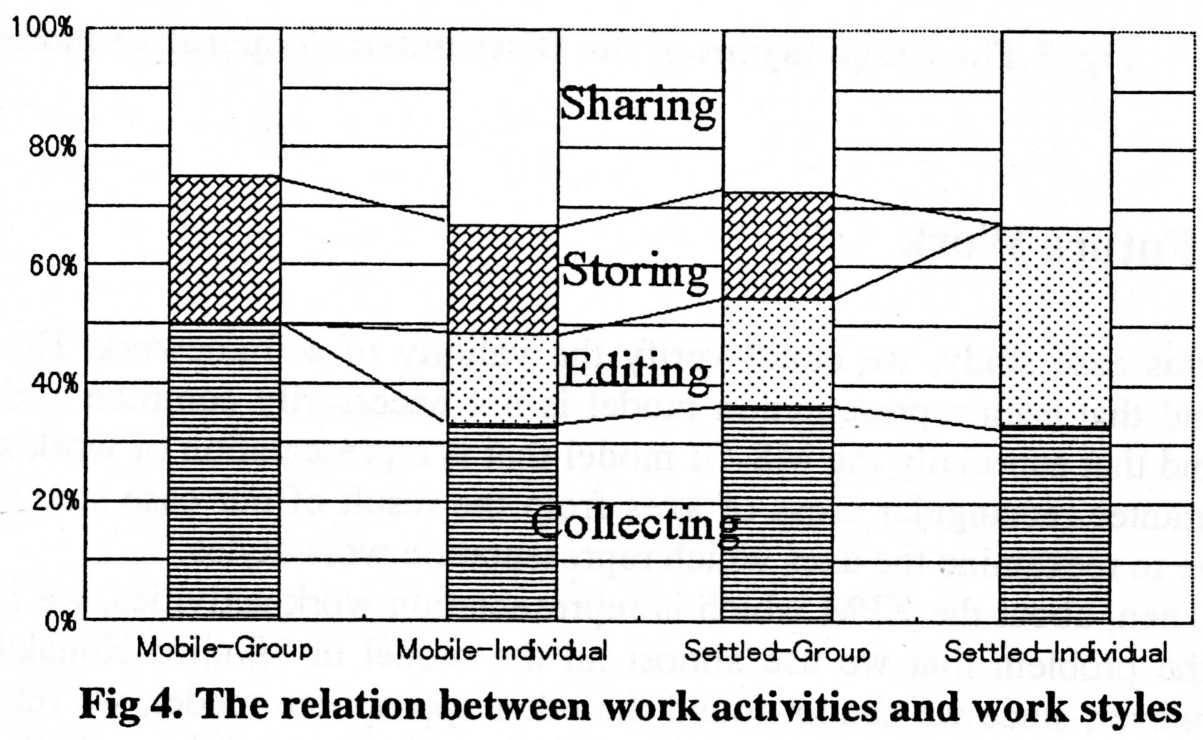

\subsection{Construction of the Distributed Cooperative model office}

We actually built a model office called the Distributed Cooperative Model Office for supporting distributed cooperative work based on our framework[5][6][11]. The purpose of constructing the model office was to prevent the decline of work efficiency that can occur in distributed environment. About 30 researchers who engaged in software development at NTT DATA participated in the model office project.

When we measured cooperative work efficiency using a subject's satisfaction rating, we found that satisfaction improved $30 \%$ in the model office. We interpret future offices should be built according to the framework's recommendations.

\section{Future works}

In this case study, we could verify the validity of a framework. However, we also found that each representation model is not necessarily common and complete. We found that especially the MS-GI model that is representation of work styles could not guarantee rectangular cross of axes from the result of our case study. Therefore, we have to reexamine the axes which representation work styles.

Then, about the KPM which is representation work activities, we recognize that it is the problem that we use almost all the model of "Ikujiro Nonaka" as he wrote. However, since we cannot found the other appropriate model, we reluctantly use the KPM as representation of work activities. While verifying whether the KPM is representing work activities truly, we have to grope for other representation models for work activities. 
About the relationship between the KPM and the SOU model, there are two problems to generalize it. The problems are that one is examining only SOU elements introduced into the model office, and another is that subject's work style is partial(strong Settle-Individual), and the number of subject is about 30 only. Therefore, we have to extend the verification range about the relation between the KPM and the SOU model in the future. Moreover, we may have not to prepare the general mapping but the mapping for every work styles. Furthermore, we have to form a still detailed hypothesis about it.

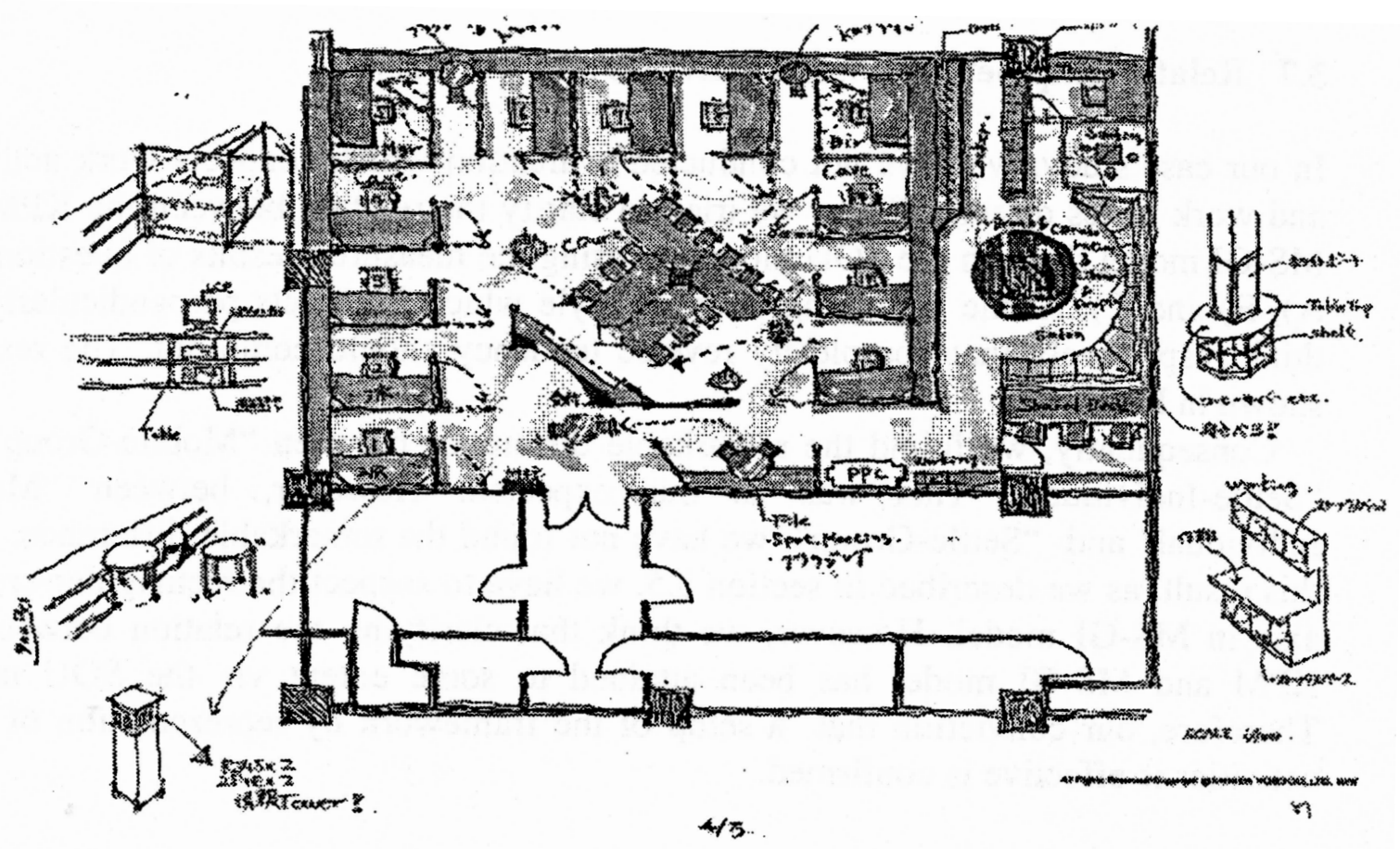

Fig. 5 The rough layout of the Distributed Cooperative model office

\section{Conclusion}

In this paper, we presented the representational models for work factors, consisting of work activities, work styles and a work environment. We have applied the framework for an organization and found that the representations are useful to identify some relationships among work factors. Certain types of work styles are dependent on determining the importance of elements of the work environment. Although the result is in some way obvious, the value of the work resides in that we could reveal the relationship based on data collected through the actual practice. The framework is found to be a promising approach to identify patterns among work factors. By using such patterns to designing work environment, we would be able to construct a useful and productive work environment by way of identifying people's work activities and styles.

\section{Acknowledgements}

This paper was strengthened by the comments of Tom Finholt, University of Michigan and from the three anonymous reviewers. We are so thank for them. We would like to thank our colleagues at NTT Data Corp for their helpful advice and cooperation in our studies. We are also very grateful Masayuki Inamochi and Takashi Nagase at Insti- 
tute of Office Productivity and Environment UCHIDA YOKO Corporation for the valuable discussions in developing the models.

\section{References}

Cross, T. B. and Raizman, M. B.: Telecommuting: The Future Technology of Work, Richard D. Irwin, Inc., 1986

DeMarco, T., Lister, T.: Peopleware: Productive Projects and Teams, Dorset House Publishing Co., Inc., 1987

Drucker, P.: The Coming of the New Organization, Harvard Business Review, Jan.-Feb. 1988, pp. 45-53, 1988

Grudin, J.: Why CSCW Applications Fail: Problems in the Design and Evaluation of Organizational Interfaces, CSCW '88 Proc. ACM, pp.85-93, 1988

Ikeda, F., Sakamaki, M., Aoki, H., Shingo, T. and Nakakoji, K.: Improving software quality from the viewpoint of knowledge production and the work environment, $6^{\text {th }}$ European Conference on Software Quality, 1999

Ikeda, F., Sakamaki, M., Aoki, H., Shingo, T. and Nakakoji, K.: Toward an environment to support distributed work by integrating information technologies, physical spaces and practice, Information Processing Society of Japan, SIG Notes, GW-24, 1997

Iivari, J.: "Why are CASE Tools not Used?", Comm. ACM, Vol.39, No.10, pp.94-103, 1996

McCue, G.: IBM's Santa Teresa Laboratory -Architecture Design for Program Development, IBM Systems Journal, Vol. 17, No.1, 1978

Mital, R. M., Kim, M. M. and Berg, R.A.: A CASE Study of Workstation Usage During the Earlry Stages of the Software Development Life Cycle, Proc. ACM Symp. on Practical Software Development Environments, pp. 70-76, 1986

Nonaka, I. and Takeuchi, H.: "The Knowledge-Creating Company", Oxford University Press, 1995

Sakamaki, M., Ikeda, F., Aoki, H.: Research for evaluation of an environment to support distributed cooperation work by integrating information technologies, physical spaces and practice, Information Processing Society of Japan, SIG Notes, GW-29, 1998

Thadhani, A. J.: Factors Affecting Programmer Productivity during Application Developments, IEEE Software, Vol.6, pp. 15-22, 1984 